\title{
Pharmaceutical quality of seven generic Levodopa/Benserazide products compared with original Madopar ${ }^{\circledR} /$ Prolopa $^{\circledR}$
}

\author{
Urs E Gasser ${ }^{1}$, Anton Fischer ${ }^{2}$, Jan P Timmermans ${ }^{2}$ and Isabelle Arnet ${ }^{3 *}$
}

\begin{abstract}
Background: By definition, a generic product is considered interchangeable with the innovator brand product. Controversy exists about interchangeability, and attention is predominantly directed to contaminants. In particular for chronic, degenerative conditions such as in Parkinson's disease (PD) generic substitution remains debated among physicians, patients and pharmacists. The objective of this study was to compare the pharmaceutical quality of seven generic levodopa/benserazide hydrochloride combination products marketed in Germany with the original product (Madopar $^{\circledR} /$ Prolopa $^{\circledR}$ 125, Roche, Switzerland) in order to evaluate the potential impact of Madopar ${ }^{\circledR}$ generics versus branded products for PD patients and clinicians.

Methods: Madopar ${ }^{\circledR} /$ Prolopa $^{\circledR} 125$ tablets and capsules were used as reference material. The generic products tested (all $100 \mathrm{mg} / 25 \mathrm{mg}$ formulations) included four tablet and three capsule formulations. Colour, appearance of powder (capsules), disintegration and dissolution, mass of tablets and fill mass of capsules, content, identity and amounts of impurities were assessed along with standard physical and chemical laboratory tests developed and routinely practiced at Roche facilities. Results were compared to the original "shelf-life" specifications in use by Roche.

Results: Each of the seven generic products had one or two parameters outside the specifications. Deviations for the active ingredients ranged from $+8.4 \%$ (benserazide) to $-7.6 \%$ (levodopa) in two tablet formulations. Degradation products were measured in marked excess (+26.5\%) in one capsule formulation. Disintegration time and dissolution for levodopa and benserazide hydrochloride at 30 min were within specifications for all seven generic samples analysed, however with some outliers.

Conclusions: Deviations for the active ingredients may go unnoticed by a new user of the generic product, but may entail clinical consequences when switching from original to generic during a long-term therapy. Degradation products may pose a safety concern. Our results should prompt caution when prescribing a generic of Madopar ${ }^{\circledR} /$ Prolopa $^{\circledR}$, and also invite to further investigations in view of a more comprehensive approach, both pharmaceutical and clinical.
\end{abstract}

Keywords: Parkinson's Disease, Levodopa, Benserazide hydrochloride, Pharmaceutical quality, Generics

\section{Background}

Parkinson's disease (PD) is a progressive neurodegenerative disorder affecting primarily dopaminergic neuronal systems. Typical manifestations include motor symptoms (bradykinesia, rigidity, tremor, gait and postural instability) and non-motor symptoms including cognitive and emotional dysfunction [1-3]. Levodopa or L-dopa is a naturally

\footnotetext{
* Correspondence: isabelle.arnet@unibas.ch

${ }^{3}$ Pharmaceutical Care Research Group, Department of Pharmaceutical Sciences, University of Basel, Klingelbergstr. 50, 4056, Basel, Switzerland Full list of author information is available at the end of the article
}

occurring amino acid (L-3,4-dihydroxyphenylalanine). It is a prodrug and the precursor of dopamine (DA), a neurotransmitter that is severely reduced in PD due to degeneration of neuronal cells $[2,3]$. Levodopa has been the standard medical therapy for PD since its discovery approximately 40 years ago [4] and is recognized as a classic example of a brain neurotransmitter substitution therapy. When given systemically, levodopa crosses the blood-brain barrier and is converted to DA by L-dopa decarboxylase (DDC) [5]. When administered orally, a high pre-systemic conversion to DA occurs in the gut by

\section{Biomed Central}

(c) 2013 Gasser et al.; licensee BioMed Central Ltd. This is an Open Access article distributed under the terms of the Creative Commons Attribution License (http://creativecommons.org/licenses/by/2.0), which permits unrestricted use, distribution, and reproduction in any medium, provided the original work is properly cited. 
the enzyme L-amino acid decarboxylase (AADC), reducing the systemic available dose of levodopa to $30 \%$ [6,7]. Levodopa is thus coadministered in a $4 / 1$ ratio with an AADC inhibitor such as benserazide hydrochloride. This combination can triple the oral bioavailability of levodopa, and markedly reduces both the required levodopa therapeutic dose and the severity of dopaminemediated gastrointestinal and cardiovascular side-effects [8]. Levodopa has a short half-life of $1.5 \mathrm{~h}$, even when coadministered with an AADC inhibitor [9]. When administered with benserazide hydrochloride, the initial dose is $100 \mathrm{mg}$ to $200 \mathrm{mg}$ levodopa daily given as levodopa $50 \mathrm{mg} /$ benserazide $25 \mathrm{mg}$ up to 2 to 4 times a day. Treatment may start at levodopa $300 \mathrm{mg}$ daily in advanced disease. The recommended maximum maintenance dose is $800 \mathrm{mg}$ levodopa daily in multiple divided doses $[9,10]$.

Levodopa is a white or almost white crystalline powder which darkens on exposure to air and light [10]. It is odourless, almost tasteless, slightly soluble in water and soluble in aqueous solutions of mineral acids and alkali carbonates [10]. Levodopa is purchased on the pharmaceutical market. Benserazide hydrochloride is a white or almost white crystalline powder, slightly soluble in ethanol, and soluble in water. Benserazide hydrochloride decomposes slowly in aqueous solution. It is synthetically produced by Roche.

Madopar ${ }^{\circledR} /$ Prolopa $^{\circledR} 125 \mathrm{mg}$ (levodopa $100 \mathrm{mg}+$ benserazide hydrochloride $25 \mathrm{mg}$ ) exists as scored tablets and capsules for Parkinson's disease [9].

By definition, a generic product is considered interchangeable with the innovator brand product and needs to demonstrate the same qualitative and quantitative composition in active substances, the same pharmaceutical form and bioequivalence with the reference product after a single dose [11]. The different salts, esters, complexes or derivatives of an active substance are considered to be the same active substance, and thus different excipients, colour agents, flavours and preservatives are allowed. Generics may also differ in characteristics such as shape, size, colour, scoring configuration, and release mechanisms [12-16]. Controversy exists about interchangeability, mainly because of the questionable validity of the current criteria the evaluation is performed in a small, young and healthy population; no clinical efficacy data are required; short term study [17]. Thus, therapeutic equivalence is not necessarily guaranteed. However, attention is predominantly directed to contaminants that could cause adverse clinical events and ultimately fatal issues [5-7]. In clinical settings, health professionals are aware that patient response and susceptibility to levodopa vary widely, especially in advanced PD, and that levodopa blood levels correlate with the emergence of many symptoms, including motor manifestations like dyskinesia and "off" periods. Thus, even small variations in levodopa availability and consequently subtle fluctuations in levodopa blood levels can trigger motor complications. Since generic formulations differ from the branded product mainly in their excipients, which may affect absorption and bioavailability [15], simple bioequivalence cannot suffice to ensure comparable clinical efficacy and safety, especially in PD.

Roche's standard operating procedures (SOPs) describe the qualitative and quantitative pharmaceutical tests for physical and chemical purity of the substance. They were accepted by regulatory authorities as part of the Madopar ${ }^{\circledR}$ registration documentation and form the basis of Mapopar ${ }^{\circledR} / \operatorname{Prolopa}^{\circledR}$ specifications in the United States, European and British Pharmacopoeias. The current investigation compares the pharmaceutical quality of seven generic products with the specifications of the original Madopar ${ }^{\circledR}$ and raises questions about safety and interchangeability.

\section{Methods}

Samples of generic levodopa/benserazide hydrochloride products, all $100 \mathrm{mg} / 25 \mathrm{mg}$ formulations, were purchased in 2011 as commercial goods in a community pharmacy in Germany and tested within their expiry dates.

\section{Pharmaceutical quality tests}

The tests were colour (tablets and capsules), colour and appearance of powder (capsules), mass of tablets and fill mass of capsules, disintegration and dissolution, content of active pharmaceutical ingredient (API), and the identity and amounts of impurities.

\section{Colour}

Tablet colour was assessed visually according to the standards described in the "Munsell Book of Color" (1996). Capsule colour was assessed by comparison to reference capsules. Madopar ${ }^{\circledR}$ specifications for colour are pale red for tablets and flesh-coloured opaque for the capsule body and powder-blue opaque for the capsule cap.

\section{Mass of tablets}

Twenty tablets were weighed and average mass was calculated. Madopar ${ }^{\circledR}$ specifications for average mass of tablets are $267-283 \mathrm{mg}(275 \mathrm{mg} \pm 3 \%)$.

\section{Fill mass of capsules}

Twenty capsules were weighed, emptied, and the empty capsules weighed; the average weight of the capsule fill mass was derived from the difference. Madopar ${ }^{\circledR}$ specifications for capsule fill mass are $142.5-157.5 \mathrm{mg}$ (150 mg $\pm 5 \%$ ). 


\section{Disintegration time}

Tablets and capsules were tested according to Ph. Eur. / USP (tablets: apparatus A without discs using water at $37^{\circ} \mathrm{C}$; capsules: apparatus A using discs and $0.1 \mathrm{M} \mathrm{HCl}$ at $37^{\circ} \mathrm{C}$ ). Madopar ${ }^{\circledR}$ specifications for disintegration time are not more than $15 \mathrm{~min}$ for tablets and not more than 30 min for capsules.

\section{Dissolution}

Dissolution was tested using the Ph. Eur. rotating basket apparatus and $0.1 \mathrm{~N} \mathrm{HCl}$ at $37^{\circ} \mathrm{C}$ as the dissolution medium. Samples were measured by UV-HPLC at $220 \mathrm{~nm}$. Madopar ${ }^{\circledR}$ shelf-life specifications for dissolution after $30 \mathrm{~min}$ are at least $75 \%$ for tablets and at least $80 \%$ for capsules.

\section{Content of levodopa, benserazide hydrochloride, related substances and impurities}

Madopar ${ }^{\circledR}$ specifications for content of active substances are levodopa $95.0-105.0 \mathrm{mg}(100 \mathrm{mg} \pm 5 \%)$ and benserazide hydrochloride $27.1-29.9 \mathrm{mg}(28.5 \mathrm{mg} \pm 5 \%)$ per unit. The content of active substances and degradation products was determined using HPLC. Benserazide is degradated by hydrolysis to Ro 04-1419, which can bind one molecule of benserazide to the dimer Ro 08-1580. The specifications for content of specific degradation products of benserazide hydrochloride are limited to $3.49 \%$ (tablets) and $0.49 \%$ (capsules). The maximum permissible amount of other impurities is set at $1.04 \%$ (tablets) and $0.54 \%$ (capsules). The specifications for other impurities are no more than $0.54 \%$ (tablets) and $0.24 \%$ (capsules) for any individual impurity.

\section{Results}

Analysis was performed according to the standard physical and chemical laboratory tests developed and routinely practiced at Roche facilities.

\section{Physical characteristics}

The physical characteristics of the tested products are shown in Table 1. None of the generic tablets was crossscored on both sides like the original product, but all had either a single break bar on both sides (Betapharm) or on one side only. The colours of all generic products were similar to Madopar ${ }^{\circledR} /$ Prolopa $^{\circledR}$ except for one capsule formulation (Teva).

\section{Mass, fill mass, disintegration time and dissolution rate}

Mass exceeded the upper limits of the specifications in five of the seven generic products (Table 2), and by almost $200 \%$ for all three capsule formulations. Disintegration times (min - max: tablets 4.30 - 6.30 min; capsules $5.40-16.10 \mathrm{~min}$ ) were within specification. Dissolution values after 30 min complied with the specifications,

Table 1 Physical characteristics of Madopar $^{\circledast} / \mathrm{Prolopa}^{\circledR}$ and seven generic products

\begin{tabular}{|c|c|c|c|c|}
\hline Brand name & Manufacturer & Colour & Appearance & \\
\hline \multicolumn{5}{|l|}{ Tablets } \\
\hline \multirow[t]{2}{*}{ Madopar } & \multirow[t]{2}{*}{ Roche Pharmaceuticals } & \multirow[t]{2}{*}{ Pale red } & \multicolumn{2}{|l|}{ Above: cross-scored } \\
\hline & & & \multicolumn{2}{|l|}{ Below: cross-scored } \\
\hline \multirow[t]{2}{*}{ Levodopa/ Benserazid beta } & \multirow[t]{2}{*}{ Betapharm Arzneimittel GmbH } & \multirow[t]{2}{*}{ Pale red, speckled } & \multicolumn{2}{|l|}{ Above: score line } \\
\hline & & & \multicolumn{2}{|l|}{ Below: score line } \\
\hline \multirow[t]{2}{*}{ Levodopa/ Benserazid-CT } & \multirow[t]{2}{*}{ CT Arzneimittel GmbH } & \multirow[t]{2}{*}{ Pale red, speckled } & \multicolumn{2}{|l|}{ Above: score line } \\
\hline & & & \multicolumn{2}{|l|}{ Below: none } \\
\hline \multirow[t]{2}{*}{ dopadura B } & \multirow[t]{2}{*}{ MYLAN dura } & \multirow[t]{2}{*}{ Pale red, speckled } & \multicolumn{2}{|l|}{ Above: score line } \\
\hline & & & \multicolumn{2}{|l|}{ Below: none } \\
\hline \multirow{2}{*}{$\begin{array}{l}\text { Levodopa/ Benserazid } \\
\text { ratiopharm }\end{array}$} & \multirow[t]{2}{*}{ ratiopharm $\mathrm{GmbH}$} & \multirow[t]{2}{*}{ Pale red, strongly speckled } & \multicolumn{2}{|l|}{ Above: score line } \\
\hline & & & \multicolumn{2}{|l|}{ Below: none } \\
\hline Capsules & & & Appearance of the content & Colour of the content \\
\hline \multirow[t]{2}{*}{ Madopar } & \multirow[t]{2}{*}{ Roche Pharmaceuticals } & Body: flesh-coloured, opaque & \multirow[t]{2}{*}{ Fine granular powder } & \multirow[t]{2}{*}{ Light beige } \\
\hline & & Cap: powder-blue, opaque & & \\
\hline \multirow[t]{2}{*}{ Levopar } & \multirow[t]{2}{*}{ HEXAL AG } & Body: flesh-coloured, opaque & \multirow[t]{2}{*}{ Fine granular powder } & \multirow[t]{2}{*}{ Brown } \\
\hline & & Cap: powder-blue, opaque & & \\
\hline \multirow[t]{2}{*}{ Levodopa comp. B STADA } & \multirow[t]{2}{*}{ STADApharm GmbH } & Body: flesh-coloured, opaque & Fine granular powder & Almost white \\
\hline & & Cap: powder-blue, opaque & & \\
\hline Levobens-Teva & TEVA GmbH & Body: midnight blue, opaque & Fine granular powder & Almost white \\
\hline & & Cap: fluorescent-pink, opaque & & \\
\hline
\end{tabular}


Table 2 Mass, content of active ingredients, and dissolution time of seven generic products compared to Madopar ${ }^{\circledR}$ Prolopa ${ }^{\circledR}$ specifications

\begin{tabular}{|c|c|c|c|c|}
\hline Brand name & Mass (mg) & Levodopa (mg) & Benserazide hydrochloride (mg) & Dissolution after $30 \mathrm{~min}$ \\
\hline Tablet specifications & $267.0-283.0$ & $95.0-105.0$ & 27.1-29.9 & $>75 \% />75 \%$ \\
\hline Levodopa/ Benserazid beta & 265 & $92.4^{*}$ & $30.9^{*}$ & $99 \%$ / 99\% \\
\hline Levodopa/ Benserazid-CT & $284^{*}$ & 98.2 & 27.8 & $101 \% / 97 \%$ \\
\hline dopadura B & 283 & $94.4^{*}$ & $26.9^{*}$ & $100 \% / 97 \%$ \\
\hline Levodopa/ Benserazide ratiopharm & $284^{*}$ & 95.5 & 28.8 & $101 \% / 98 \%$ \\
\hline Capsule specifications & Fill mass (mg) 142.5-157.5 & $95.0-105.0$ & 27.1-29.9 & $>80 \% />80 \%$ \\
\hline Levopar & $298.6^{*}$ & 98.3 & 27.5 & $80 \% / 84 \%$ \\
\hline Levodopa comp. B STADA & $299.0^{*}$ & 98.7 & 27.6 & $100 \% / 96 \%$ \\
\hline Levobens-Teva & $222.3^{*}$ & 99.5 & 28.3 & $89 \% / 86 \%$ \\
\hline
\end{tabular}

* Values marked with an asterisk indicate deviations from Roche specifications.

however, some single values were below specification (Levobens-Teva 16\%, 25\%; Levopar 68\%, 49\%, 72\% 78\%), indicating large variations in dissolution properties for single capsules.

\section{Content of active substances, degradation products, and} impurities

Content requirements for the active substances were unmet in two of the four tablet formulations, with content of levodopa below the limits, and content of benserazide hydrochloride below and above the limits (Table 2). All products contained impurities, exceeding the limits by $79 \%$ in one generic product (Table 3 ).

\section{Discussion}

Comparison of the pharmaceutical quality of Madopar ${ }^{\circledR} /$ Prolopa ${ }^{\circledR}$ with seven generic products showed that at least one of the tested parameters fell outside Roche shelflife specifications in all products tested and identified two areas of concern: Content of active substances and impurities. Since even small variations of levodopa availability and consequently subtle fluctuations in levodopa blood levels can trigger motor complications, the observed outof-specification values could suffice to unbalance symptom management, especially in stabilized patients. In switching to a new formulation, patients may face weeks of complex retitration and further visits to their neurologists. In 2010-11 the worldwide shortage of branded Sinemet (carbidopa-levodopa), followed by shortage of its generic formulation (all formulations and all dosages), revealed the distress of the Parkinson community through the testimony of thousands of patients forced to switch to generics [18]. Patients uniformly reported a negative experience and several clinical issues, e.g. slower onset of effect, faster waning of effect, dose adjustment to compensate for the decreased medication effect, symptom exacerbation (e.g. dyskinesia), and side effects such as poorer sleep quality and increased impulsivity. No patient reported a preference for the generic version. Replacement medication was perceived as less effective, probably due to patients receiving differing generics at each renewal, thus getting fluctuations in blood levels with every new product.

The development of a branded formulation requires the assessment of pharmacokinetic parameters in healthy

Table 3 Identity and amounts of related substances and impurities in seven generic products compared to Madopar ${ }^{\circledR}$ Prolopa ${ }^{\circledR}$ specifications

\begin{tabular}{|c|c|c|c|c|c|}
\hline Degradation product & Ro 04-1419 & Ro 08-1580 & Ro $04-1419$ + Ro 08-1580 & Others each & Others total \\
\hline Tablets Upper limits (\%) & 1.54 & 2.49 & 3.49 & 0.54 & 1.04 \\
\hline Levodopa/ Benserazid beta & 0.35 & 0.47 & 0.83 & $0.19,0.09$ & 0.28 \\
\hline Levodopa/ Benserazid-CT & 0.28 & 0.48 & 0.75 & $0.09,0.08$ & 0.17 \\
\hline Levodopa/ Benserazide ratiopharm & 0.25 & 0.37 & 0.62 & $0.16,0.05$ & 0.21 \\
\hline dopadura B & 0.26 & 0.34 & 0.60 & 0.12 & 0.12 \\
\hline Capsules Upper limits (\%) & 0.54 & 0.54 & 0.49 & 0.24 & 0.54 \\
\hline Levopar & 0.12 & 0.34 & 0.46 & 0.10 & 0.10 \\
\hline Levodopa comp. B STADA & 0.16 & 0.32 & 0.49 & $0.11,0.11$ & 0.11 \\
\hline Levobens-Teva & 0.36 & 0.25 & $0.62^{*}$ & $0.10,0.12,0.10$ & 0.33 \\
\hline
\end{tabular}

*Values marked with an asterisk indicate deviations from Roche specifications. 
subjects, and a clinical study program to proof efficacy, safety and tolerability in the target patient population. Market authorisation for generic equivalents, however, requires only the documentation of bioequivalence with branded counterparts in healthy subjects, using one lot of branded product, and without accounting for country to country differences $[19,20]$. Some small studies $[21,22]$ suggested that the generic formulation of carbidopalevodopa given in a single dose to PD patients was bioequivalent to brand Sinemet. However, the same authors report clinical worsening with marked motor fluctuations in a long-term open-label study following conversion to generic carbidopa-levodopa [21]. Approval by the authorities is based on the assumption that demonstrating bioequivalence in pharmacokinetic studies in healthy volunteers suffices to demonstrate similar tolerability and efficacy in patients. Differences in the excipients used for generic formulations and the presence of impurities which may affect both the absorption and bioavailability of active ingredients in patients create a potential risk of "relative therapeutic in-equivalence" [15]. Furthermore, the intra-individual peculiarity of PD patients, such as slow absorption of the first orally administered dose of medication in the morning due to low gastric motility [23] makes the variability of blood concentrations from generic drugs unpredictable. Furthermore, PD patients often use multiple drugs e.g., dopamine agonists, monoamine oxydase inhibitors, anticholinergics, and psychotropics. Since issues regarding drug-drug interactions are not addressed by bioequivalence studies, potential risks cannot be excluded [1,23]. Unpredictable blood concentrations also expose patients to a higher risk of concentrationdependent drug-drug interactions. In addition, unknown excipients and impurities may trigger allergic reactions or even intolerance $[24,25]$. Score lines are a further area of concern. Madopar ${ }^{\circledR} /$ Prolopa $^{\circledR}$ is crossscored for easy splitting, since many patients commonly split their tablets to adjust their doses. Easy splitting of the medication for optimal individual dosing is a critical patient-care requirement. Without score lines, or with only one score line on one side, as in three of the test generic tablets, tablet splitting usually requires the use of a sharp blade, which is a clear safety issue for a person with PD.

\section{Conclusions}

In conclusion, these results demonstrate areas of concern in the pharmaceutical quality of generic products, such as the content of active substances, and the composition and amount of impurities. The potential risks of "relative therapeutic in-equivalence," drug-drug interactions, and allergic reactions or intolerance should prompt caution when prescribing a generic product of
Madopar ${ }^{\circledR} /$ Prolopa $^{\circledR}$, in particular in highly susceptible PD patients with co-morbidities requiring comedication. We recommend considering the substitution of Parkinson's medication as a change in medication, requiring guidance and supervision by the patient's physician. Any generic can be used to initiate first treatment. It will be effective and less expensive than the branded product, but will need a similar process of titration until symptom control is achieved. The challenge for patient, prescribing physician and pharmacist will be to ensure that the same generic formulation is dispensed at each refill. Switching back and forth between brand and generic, or even between generics, is a recipe for problems that may cancel out the savings achieved with the cheaper drug. Ultimately, it is the responsibility of the physician-patient-pharmacist triad to arrive at the best choice for the best patient outcome.

\section{Abbreviations}

AADC: L-amino acid decarboxylase; API: Active pharmaceutical ingredient; DA: Dopamine; DDC: L-Dopa decarboxylase; $\mathrm{HCl}$ : Hydrochloride;

L-dopa: Levodopa; PD: Parkinson's Disease.

\section{Competing interests}

FA and JPT are employees at Roche. UA and IA received consultant fees.

\section{Authors' contributions}

FA and JPT conceived and designed the study, and collected the data. UA and IA analysed and interpretated the data. All authors were involved in drafting and revising the manuscript critically for intellectual content and gave final approval of the version to be published.

\section{Author's information}

$\mathrm{UA}$ is an independent senior scientist working for different companies as a consultant. IA is a researcher at the University of Basel specialised in adherence to medication and in guidelines developement for community pharmacies. She is a lecturer and member of several pharmacy and pharmacology societies.

\section{Author details}

${ }^{1}$ ClinResearch Ltd, Aesch, Switzerland. ${ }^{2}$ F. Hoffmann-La Roche Ltd, Basel, Switzerland. ${ }^{3}$ Pharmaceutical Care Research Group, Department of Pharmaceutical Sciences, University of Basel, Klingelbergstr. 50, 4056, Basel, Switzerland.

Received: 27 December 2012 Accepted: 10 April 2013 Published: 23 April 2013

\section{References}

1. Schapira AHV, Olanow CE, eds: Principles of treatment in Parkinson's disease. Philadelphia, PA: Elsevier Health Sciences; 2005.

2. Fahn S, Sulzer D: Neurodegeneration and neuroprotection in Parkinson disease. NeuroRx 2004, 1:139-154.

3. Sulzer D: Multiple hit hypotheses for dopamine neuron loss in Parkinson's disease. Trends Neurosci 2007, 30(5):244-250.

4. Yahr MD, Duvoisin RC, Schear MJ, Barrett RE, Hoehn MM: Treatment of Parkinsonism with levodopa. Arch Neurol 1969, 4:343-354.

5. Hauser RA, Zesiewicz TA: Advances in the pharmacologic management of early Parkinson disease. Neurologist 2007, 13(3):126-132.

6. Jankovic J, Stacy M: Medical management of levodopa-associated motor complications in patients with Parkinson's disease. CNS Drugs 2007, 21(8):677-692.

7. Esposito E, Cuzzocrea S: New therapeutic strategy for Parkinson's and Alzheimer's disease. Curr Med Chem 2010, 17(25):2764-2774.

8. Contin M, Martinelli P: Pharmacokinetics of levodopa. J Neurol 2010, 257(Suppl 2):S253-S261. 
9. Swiss Summary of product characteristics of Madopar ${ }^{\circledR} / \mathrm{Prolopa}^{\circledR}$. www. swissmedicinfo.ch>Madopar.

10. Reynolds JEF (Ed): Martindale - the Extra Pharmacopoeia. 30th edition. London: The Pharmaceutical Press; 1993.

11. EU/1/98/071/001-006 - Type II Variation EMEA/H/C/00154/I/0045: Widening of dissolution specification Q-value at shelf-life from $75 \%$ to $65 \%$. 2007. www.ema.europa.eu/docs/en_GB/document_library/Scientific_guideline/ 2010/01/WC500070039.pdf.

12. Duh MS, Cahill KE, Paradis PE, Cremieux PY, Greenberg PE: The economic implications of generic substitution of antiepileptic drugs: a review of recent evidence. Expert Opin Pharmacother 2009, 10(14):2317e28.

13. Berg MJ, Gross RA, Haskins LS, Zingaro WM, Tomaszewski KJ: Generic substitution in the treatment of epilepsy: patient and physician perceptions. Epilepsy Behav 2008, 13(4):693e9.

14. Andermann F, Duh MS, Gosselin A, Paradis PE: Compulsory generic switching of antiepileptic drugs: high switchback rates to branded compounds compared with other drug classes. Epilepsia 2007, 8(3):464-469.

15. Go C, Rosales RL, Schmidt P, Lyons KE, Pahwa R, Okun MS: A generic versus branded pharmacotherapy in Parkinson's disease: Does it matter? A review. Parkinsonism Relat Disords 2011, 17:308-312.

16. Ferner RE, Lenney W, Marriott JF: Controversy over generic substitution. BMJ 2010, 340:C2548.

17. Borgheini $\mathrm{G}$ : The bioequivalence and therapeutic efficacy of generic versus brand-name psychoactive drugs. Clin Ther 2003, 25(6):1578-1592.

18. National Parkinson Foundation: Lessons from the 2011 Sinemet shortageThe National Parkinson Foundation's Helpline speaks. 2012. Available from: www.parkinson.org [March 15, 2013] www.parkinson.org/ NationalParkinsonFoundation/files/ae/aea8377b-cf22-46d0-bdc63922290efb60pdf.

19. Meredith P: Bioequivalence and other unresolved issues in generic drug substitution. Clin Ther 2003, 5(11):2875e90

20. US Food and Drug Administration: Guidance for industry: Bioavailability and bioequivalence studies for orally administered drug products general consideration. [July 18, 2010]. 2010. Available from: www.fda. gov/cder/guidance/4964dft.pdf.

21. Pahwa R, Marjama J, McGuire D, Lyons K, Zwiebel F, Silverstein P, et al: Pharmacokinetic comparison of Sinemet and Atamet (generic carbidopa/levodopa): a single-dose study. Mov Disord 1996, 11:427-430.

22. Chaná P, Fierro A, Reyes-Parada M, Sáez-Briones P: Pharmacokinetic comparison of Sinemet and Grifoparkin (levodopa/carbidopa 250/ $25 \mathrm{mg}$ ) in Parkinson s disease: a single dose study [Article in Spanish]. Rev Med Chil 2003, 131:623-631.

23. Fernandez N, Garcia JJ, Diez MJ, Sahagun AM, Gonzalez A, Diez R, et al: Effects of slowed gastrointestinal motility on levodopa pharmacokinetics. Auton Neurosci 2010, 156(1-2):67e72.

24. Sims-McCallum RP: Adverse reaction caused by excipients in mercaptopurine tablets. Ann Pharmacother 2007, 41(9):1548.

25. Hebron $\mathrm{BS}$, Hebron $\mathrm{HJ}$ : Aspirin sensitivity: acetylsalicylate or excipients, Intern Med 2009, 39(8):546-549.

\section{Submit your next manuscript to BioMed Central and take full advantage of:}

- Convenient online submission

- Thorough peer review

- No space constraints or color figure charges

- Immediate publication on acceptance

- Inclusion in PubMed, CAS, Scopus and Google Scholar

- Research which is freely available for redistribution 